\title{
Écologie et économie des interactions entre filières agricoles et territoire : quels concepts et cadre d'analyse ?
}

\author{
Sophie Madelrieux ${ }^{1, *}$, Nicolas Buclet ${ }^{2}$, Philippe Lescoat ${ }^{3}$ et Marc Moraine ${ }^{4}$ \\ ${ }^{1}$ Université Grenoble Alpes, IRSTEA, UR DTGR, 2 rue de la papeterie, BP76, 30402 St Martin d'Hères Cedex, France \\ ${ }^{2}$ Université Grenoble Alpes, IUG, 14 Avenue Marie Reynoard, 38100 Grenoble, France \\ 3 AgroParisTech, 16 rue Claude Bernard, 75231 Paris Cedex 05, France \\ ${ }^{4}$ ISARA-Lyon, 23 rue Jean Baldassini, 69364 Lyon cedex 07, France
}

\begin{abstract}
Résumé - Les filières agricoles connaissent une importante restructuration, se traduisant par une déconnexion de plus en plus forte entre opérateurs des filières et ceux des territoires, et entre lieux de production et usages des ressources locales. Les filières utilisent des ressources des territoires, mais n'y opèrent souvent qu'une partie de leur activité, et ne se soucient pas toujours de leur impact local, environnemental comme socioéconomique. Des acteurs de territoires, porteurs quant à eux d'enjeux de développement socioéconomique et environnementaux, sont inquiets des incidences de ces transformations et s'interrogent sur la possibilité de re-territorialiser certaines filières. Pour alimenter les réflexions de ces acteurs, nous cherchons à mieux comprendre les interactions entre filières agricoles et territoires, notamment les interactions entre les flux générés, les écosystèmes, et les systèmes d'acteurs qui les pilotent. L'écologie territoriale, champ de recherche interdisciplinaire, vise à mieux comprendre les modalités des interactions entre sociétés et environnement, notamment les relations entre la structuration des flux (matériels et énergétiques) et l'organisation sociale, politique, économique à l'échelle de territoires, à partir de la notion de métabolisme. Nous présentons ici une exploration de ce champ de recherche, ainsi que des champs de recherche affiliés, par des chercheurs en agronomie, en dialogue avec un chercheur en écologie territoriale. Nous explicitons les notions et présentons ce que nous en avons retenu pour élaborer un cadre d'analyse des interactions entre filières et territoires, s'appuyant sur les notions d'ancrage, de dépendance et d'empreinte. Nous discutons des intérêts et limites de l'approche dans le champ de l'agronomie et à l'échelle des territoires.
\end{abstract}

Mots clés : filière / territoire / flux / secteur agricole / écologie

\begin{abstract}
Ecology and economy of the interactions between agricultural sectors and territories: what concepts and framework of analysis. The agricultural value chains are facing an important restructuring, which translates in a more and more important disconnection between operators of the supply chains and those of the territories, and between places of production and uses of the local resources. Supply chains use local resources, but often operate only a part of their activity there, and do not always care about their local impact, either environmental or socioeconomic. Actors of territories, as environmental and socioeconomic stakeholders, are worried about incidences of these transformations and question the possibility to re-localize parts of the value chains. To feed the reflections of local actors, we seek to better understand the interactions between agricultural value chains and territories, in particular the interactions between the generated flows, the ecosystems, and the systems of actors, which pilot them. Territorial ecology, an interdisciplinary research field, aims at understanding modalities of interactions between societies and environment, and especially relations and correlations between flows (materials and energy) and the social, political, and economic organization at territorial scale, building upon the notion of metabolism. We present here an exploration of this research field by researchers in agronomy, in dialogue with a researcher in territorial ecology. We clarify the notions, and we present what we held to develop an analytical framework of the interactions between supply chains and
\end{abstract}

\footnotetext{
* Auteur de correspondance : sophie.madelrieux@irstea.fr
} 
territories, building on the notions of embeddedness, dependency, and footprint. We discuss the interests and limits of such an approach in the fields of agronomy and at a territorial level.

Keywords: supply chain / territory / flow / agricultural sector / ecology

\section{Introduction: les interactions entre filières agricoles et territoires en question}

Les filières agricoles connaissent une importante restructuration. On observe une concentration des opérateurs, l'orientation par l'aval des filières (opérateurs de la transformation et de la distribution en lien avec la demande et le marché mondial), et l'éloignement entre les centres de décisions et les lieux de production (Rastoin et Ghersi, 2010). Cela se traduit par une déconnexion de plus en plus forte entre les opérateurs des filières et ceux des territoires (Manouvrier, 2008), et entre lieux de production et usages des ressources locales (Buclet, 2011). Cela est lié à un recours accru à des intrants pour la production (Fader et al., 2013) et à l'introduction de "paquets techniques» indépendamment des questions de disponibilités, localement, des ressources nécessaires à leur mise en œuvre, ou n'incluant pas la possibilité d'utiliser des ressources disponibles localement. Ces transformations affectent les écosystèmes locaux, notamment par un accroissement des flux (entrants comme sortants) de matières et d'énergie et l'absence de bouclage des cycles de la matière (Nesme et Withers, 2016). Elles affectent aussi la vie économique et sociale des territoires. En effet, on observe globalement que l'autonomie et la souveraineté des territoires se réduisent face aux marchés agricoles et aux entreprises de l'agro-industrie (Van der Ploeg, 2008). Des acteurs de territoires (élus, chargés de développement territorial, associations), porteurs d'enjeux de développement socioéconomique et d'enjeux environnementaux, sont inquiets des incidences de ces transformations pour leurs territoires, malgré le développement de formes alternatives d'agriculture (biologique, agroécologique, etc.), s'inscrivant davantage dans des systèmes alimentaires locaux ou dans une économie circulaire (Duru et al., 2016).

Pour alimenter les réflexions des acteurs des territoires, nous cherchons au préalable à mieux comprendre les interactions entre filières agricoles et territoires, vus comme des systèmes socioécologiques (McGinnis et Ostrom, 2014). La plupart des travaux sur les relations entre filières et territoires traitent des systèmes agroalimentaires localisés (Muchnik et al., 2008) ou des filières sous indication géographique (Paus et Reviron, 2010). Mais la dimension environnementale est relativement absente de ces travaux. Pour les filières longues standard, peu de travaux abordent la structure organisationnelle de ces filières comme verrou ou levier à une (re)-territorialisation des productions agricoles (Fares et al., 2012). De plus, les approches biophysiques et socioéconomiques restent, malgré quelques tentatives, largement dissociées (Buclet, 2015). L'enjeu est de parvenir à mieux prendre en compte les interactions entre les flux générés, les écosystèmes, et les systèmes d'acteurs qui les pilotent, ce qui nécessite un pluralisme conceptuel (Costanza, 1989). Nous (chercheurs en sciences agronomiques, particulièrement intéressés par l'évolution des activités d'élevage) nous sommes tournés vers l'écologie territoriale pour traiter ces questions. En effet, l'objet de l'écologie territoriale est précisément l'étude des interactions entre les activités humaines et les systèmes socioéconomiques et écologiques dans lesquels elles sont enchâssées. Ce champ de recherche interdisciplinaire s'inscrit en filiation d'autres (écologie urbaine, bioéconomie et écologie industrielle). Comme pour tout travail interdisciplinaire, l'interconnaissance nécessite un décryptage des paradigmes et concepts. Nous présentons ici l'exploration que nous avons faite du champ de l'écologie territoriale, et des champs de recherche affiliés. Nous explicitons les notions, du point de vue de chercheurs en sciences agronomiques, en dialogue avec un chercheur en écologie territoriale. Nous présentons ce que nous en avons retenu pour élaborer un cadre d'analyse des interactions entre filières et territoires. Dans un autre article de ce même numéro (Madelrieux et al., 2017), nous mobilisons ce cadre pour réaliser une revue des méthodes existantes pour traiter les différentes dimensions des interactions entre filières agricoles et territoires.

\section{Différentes notions cherchant à relier économie et écologie des activités humaines}

L'appropriation générale de l'enjeu de durabilité a conduit à l'usage d'une multiplicité de notions cherchant à relier économie et écologie des activités humaines: économie circulaire, écologie industrielle et territoriale, bioéconomie, ou encore agroécologie en ce qui concerne plus spécifiquement les activités agricoles. Leurs définitions, quand elles sont explicitées, peuvent être variées (Schaller, 2013), et avoir évolué dans le temps. Nous proposons d'expliciter ces notions, de notre point de vue, en précisant leurs origines et principes fondateurs, leur filiation (synthétisée schématiquement sur la Fig. 1), en quoi elles se distinguent, comment elles sont utilisées et parfois détournées dans leur application par rapport aux principes initiaux. Nous nous appuyons sur une revue de la littérature, qui retrace notamment leur historique.

\subsection{Bioéconomie et ecological economics}

Les questions d'économie et d'écologie ont été longtemps traitées séparément (Diemer et Labrune, 2007). GeorgescuRoegen serait le premier à avoir posé le problème de l'économie de l'espèce humaine dans son contexte écologique global (Grinevald, 1993). C'est le fondateur de l'école de bioéconomie. Pour ce courant, la pensée économique doit retrouver son inspiration première, qui se situait historiquement au voisinage des sciences de la vie et de la physique. Les fondements mécanistes de l'économie sont abandonnés au profit de la thermodynamique, qui permet de rendre compte de l'interaction entre les processus biologiques ou économiques, et l'environnement matériel. Elle stipule notamment que l'entropie d'un système fermé ne peut que croître. La seconde loi de la thermodynamique révèle alors la dimension irréversible du processus économique qui tend à dissiper les ressources matérielles et énergétiques sur lesquelles se fonde la 


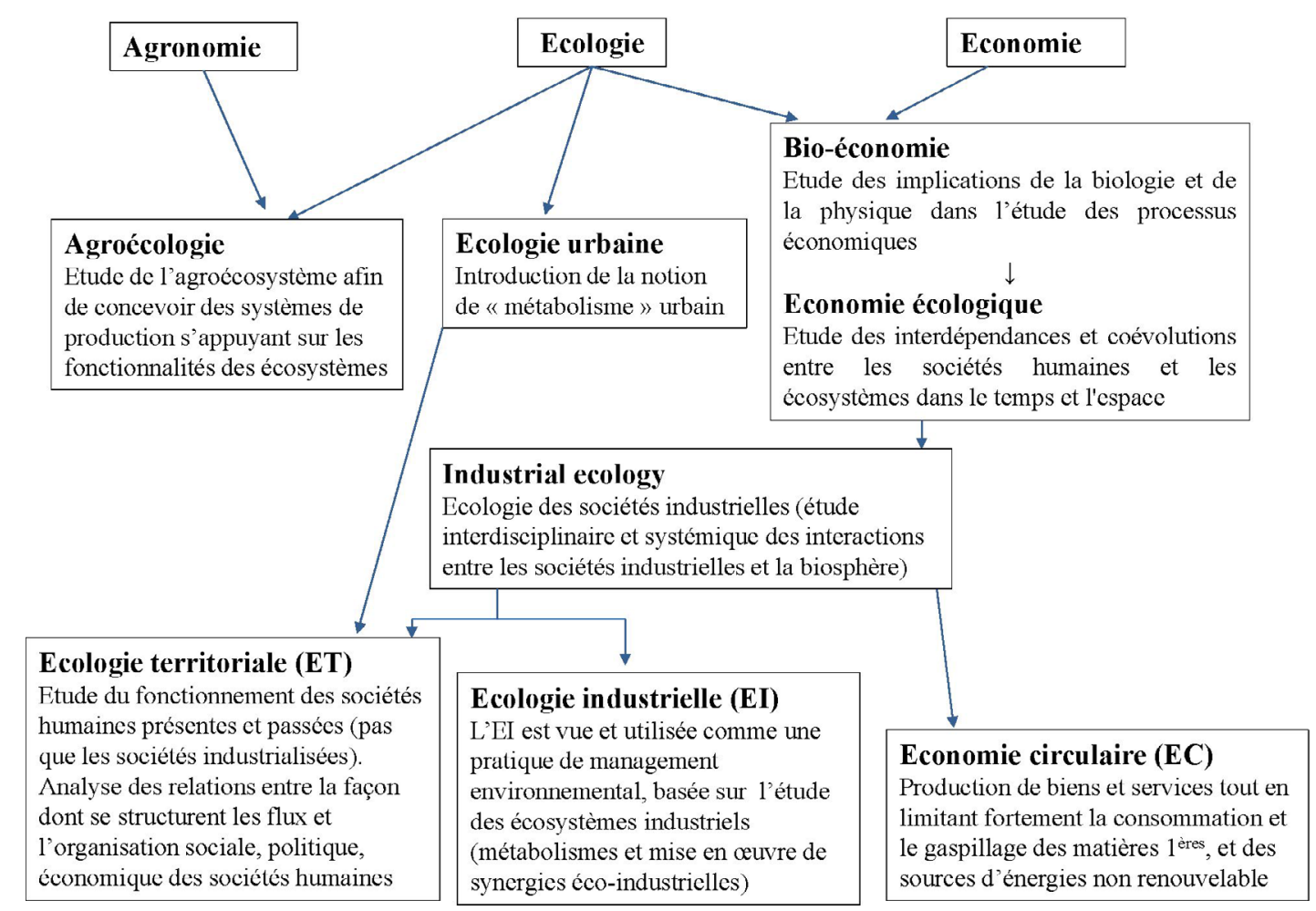

Fig. 1. Représentation schématique des filiations entre les notions cherchant à relier économie et écologie des activités humaines.

Fig. 1. Schematic representation of the filiations between the notions trying to connect economy and ecology of the human activities.

prospérité des sociétés modernes. C'est parce qu'ils ne peuvent se détacher des lois de la nature que les processus économiques transforment de manière irréversible la matière et l'énergie (Missemer, 2013). Ce courant considère le développement techno-économique de l'espèce humaine dans son enracinement biophysique, sans jamais perdre de vue les contraintes et les limites de la planète Terre (Georgescu-Roegen, 1979). Il entend donc rapprocher l'économie et l'écologie, ce qui en fait l'une des sources de l'ecological economics (économie écologique), qui vise quant à elle à étudier les interdépendances et coévolutions entre les sociétés humaines et les écosystèmes dans le temps et l'espace. Selon Costanza et al. (2015): « it is intended to be a new approach to both ecology and economics that recognizes the need to make economics more cognizant of ecological impacts and dependencies; the need to make ecology more sensitive to economic forces, incentives, and constraints; and the need to treat integrated economic-ecologic systems with a common (but diverse) set of conceptual and analytical tools ». Une des questions centrales est celle des limites du système imbriquant variables économiques, démographiques et environnementales (Meadows et al., 1972). Un certain nombre d'indicateurs sont mis au point pour mesurer l'impact des activités humaines et les conditions écologiques pour un développement socioéconomique durable, comme la dette écologique (Emelianoff, 2008) ou l'empreinte écologique (Rees, 1992).

Le terme bioéconomie a vraisemblablement été utilisé pour la première fois vers 1925 par le biologiste T.I. Baranoff pour désigner l'analyse économique de certains phénomènes biologiques. Depuis, il désigne une multiplicité de théories et de pratiques. Il peut s'agir de celle développée par
Georgescu-Roegen à partir du milieu des années 1970, ou encore du sens donné par la Commission européenne, non plus d'un champ scientifique mais d'un secteur d'activités, qui utilise la production primaire des continents et des océans pour produire de la nourriture, des matériaux et de l'énergie. La bioéconomie est alors identifiée comme une alternative majeure à une économie basée sur l'exploitation des ressources fossiles, et donne lieu à une stratégie européenne adoptée en 2012: «Innovating for sustainable growth: a bioeconomy for Europe» (European Commission, 2012).

\section{2 Écologie industrielle}

C'est dans le contexte de la bioéconomie et de l'ecological economics qu'émerge l'industrial ecology dans les années 1980. Cette dernière s'intéresse, à partir d'une vision globale et systémique, aux interactions entre les sociétés industrielles (et pas seulement le secteur industriel) et la biosphère. L'écologie industrielle aspire à fonder un nouveau paradigme dans la relation de l'homme à son environnement, en partant de l'analogie avec le fonctionnement des écosystèmes. Il s'agit de découpler la croissance économique de celle de l'utilisation des ressources, de passer d'une économie linéaire, avec des ressources extraites de l'écosystème, exploitées et retournées à l'écosystème sous forme dégradée, à une économie circulaire, ne puisant que marginalement dans les ressources naturelles afin de répondre aux besoins humains (Buclet, 2011). Par une réorganisation des activités humaines et de nouvelles formes de coopération entre acteurs, l'objectif est de rendre possible ce découplage. Elle considère que le métabolisme des activités humaines et notamment économiques peut être analysé sur le 
même modèle que celui des organismes vivants, et que son étude constitue le préalable de toute démarche en écologie industrielle (Diemer et Labrune, 2007). Il s'agit de comprendre la dynamique des flux et des stocks de matière et d'énergie liée aux activités humaines. Buclet (2015) propose une lecture historique de ce champ disciplinaire et montre que l'écologie industrielle est rapidement devenue un champ dominé par une vision d'ingénieur comme celle de Frosch et Gallopoulos (1989). L'idée centrale de découplage entre croissance économique et consommation de ressources est réduite à une réflexion sur la façon de boucler les flux de matière et d'énergie et à des questions d'optimisation et de technologie. L'écologie industrielle s'inscrit alors davantage dans une logique d'actions que de connaissance. Bien que critiquée (Ehrenfeld, 2004a), cette vision dominante s'impose, notamment aujourd'hui en France dans l'esprit de nombreux acteurs, et est restreinte à son principal champ d'investigation: les symbioses éco-industrielles (cas emblématique de Kalundborg au Danemark). Pourtant des travaux s'intéressent à la question des acteurs, des coordinations et du développement territorial (Buclet, 2015).

\section{3 Écologie territoriale}

En raison de la connotation française du terme « industriel», et $\mathrm{du}$ resserrement de sa signification, le terme «écologie territoriale » est apparu en France, afin de mettre l'accent sur une pluralité d'acteurs, d'espaces de déploiement et d'enjeux, que ces derniers soient industriels, urbains ou agricoles (Barles, 2011; Buclet, 2011). L'objectif est de se concentrer sur un territoire, plus que sur une ressource, un site ou un produit. L'écologie territoriale s'inscrit en filiation de la version initiale de l'écologie industrielle, mais aussi de l'écologie urbaine (Emelianoff, 2000) qui la première a utilisé la notion de «métabolisme» pour appréhender les problèmes métaboliques urbains (les flux de matière, d'énergie, et de déchets). Elle s'intéresse aux fonctionnements des sociétés humaines présentes et passées et pas seulement aux sociétés industrialisées (Buclet, 2015). L'écologie territoriale est un champ de recherche interdisciplinaire, dont l'objectif est de mieux comprendre les modalités des interactions entre sociétés et environnement. Elle vise à comprendre les relations et corrélations entre la façon dont se structurent les flux (matériels et énergétiques du métabolisme territorial) et l'organisation sociale, politique, économique des sociétés humaines. Le territoire est pensé comme un système d'interactions socioécologiques (McGinnis et Ostrom, 2014). Des flux matériels et immatériels circulent vers/au sein/depuis le système territorial, découpage artificiel, mais raisonné, d'un morceau de biosphère. L'écologie territoriale vise également à mieux saisir les interdépendances à d'autres territoires, et les impacts des modes de production, de consommation, de vie au sein du territoire sur son environnement, proche ou éloigné (via l'analyse des flux notamment).

À un niveau international, les travaux initiés en France ne trouvent pas d'équivalents. Il existe néanmoins des proximités fortes avec les travaux sur le métabolisme social, socioéconomique ou socioécologique selon les auteurs (FischerKowalski et Haberl, 2007; Erb, 2012; Pauliuk et Hertwich, 2015). Pour ces derniers auteurs, le concept de socioeconomic metabolism, qui a été développé dans les analyses de flux de matières et leur comptabilité, est un objet-frontière qui peut servir de paradigme pour étudier les bases biophysiques du fonctionnement des sociétés humaines. Mais l'écologie territoriale s'en écarte en positionnant le curseur tout autant du côté des sciences humaines et sociales que biophysiques. Dans la littérature se trouve aussi la notion d'écologie industrielle et territoriale en lien avec la vision opérationnelle de l'écologie industrielle, mais qui, selon nous, vise à mettre davantage l'accent sur la prise en considération des jeux d'acteurs et de leurs modes de coordination, que ne le fait l'écologie industrielle aujourd'hui (Brullot et al., 2014).

\section{4 Économie circulaire}

Le concept d'économie circulaire est étroitement lié à celui d'écologie industrielle. Erkman (2004) indique qu'un des enjeux de l'écologie industrielle est de tendre vers une économie circulaire. La traduction d'écologie industrielle dans les langues asiatiques s'apparente davantage au terme d'économie circulaire, ce qui a incité certains auteurs, comme Dominique Bourg, à ne plus se référer à l'écologie industrielle mais à l'économie circulaire. L'économie circulaire vise à promouvoir un système économique et industriel sobre en énergie et ressources naturelles pas, peu, difficilement, lentement ou coûteusement renouvelables. C'est un concept qui renvoie, selon l'Ademe (2014), à un « système économique d'échange et de production qui, à tous les stades du cycle de vie des produits (biens et services), vise à augmenter l'efficacité de l'utilisation des ressources et à diminuer l'impact sur l'environnement tout en développant le bien-être des individus ». La notion d'économie circulaire a été institutionnalisée en France à l'occasion du Grenelle de l'environnement en 2007. S'en sont suivies, en 2012, la création de l'Institut d'économie circulaire, et en 2014, l'adoption d'un texte de loi sur la transition énergétique, qui comporte un titre entièrement consacré à l'économie circulaire. Des institutions françaises comme l'Ademe ou le ministère en charge de l'environnement se saisissent de différentes approches (écologie industrielle dans sa vision restrictive, économie de la fonctionnalité, agroécologie...) pour en faire des composantes opérationnelles de l'économie circulaire. Mais il n'existe pas de communauté de recherche structurée dans le champ de l'économie circulaire. Ce qui marque une césure entre l'appropriation des concepts par les institutions et les acteurs de terrain, et les travaux menés par des communautés de recherche sur ces mêmes concepts (Buclet, 2015).

\subsection{Agroécologie}

Concernant plus spécifiquement les liens entre activités agricoles et écosystèmes, est apparu à partir des années 1930 le champ de l'agroécologie, au carrefour de l'écologie et de l'agronomie. La première utilisation du terme agroécologie renvoie à l'utilisation de méthodes écologiques au service de la recherche sur les plantes (Schaller, 2013). Un ouvrage entier lui est consacré dans les années 1960 avec des analyses croisées des interactions entre éléments biologiques (écologie) et pratiques agricoles (agronomie). À partir des années 1980, on observe une inflation des travaux scientifiques en agroécologie, incluant aussi les sciences sociales, et abordant 
aussi bien l'exploitation agricole, le paysage, le territoire que le système alimentaire (Wezel et al., 2009). Ces auteurs illustrent cette évolution, en comparant la définition de l'agronome français Hénin (1967) : «l'écologie appliquée à la production végétale et à la gestion des terres agricoles », à celle de Francis et al. (2003): "l'étude intégrée de l'écologie du système alimentaire dans son ensemble, comprenant ses dimensions écologiques, économiques et sociales, ou plus simplement l'écologie des systèmes alimentaires »).

L'agroécosystème est l'objet d'étude, dans laquelle les flux géochimiques, les transformations d'énergie, les processus biologiques et les relations sociales et économiques doivent s'analyser comme un tout, de manière interdisciplinaire (Altieri, 1987). Le cadre d'analyse des services écosystémiques (MEA, 2005) est fortement mobilisé pour développer une approche fonctionnelle des relations entre pratiques agricoles et écosystèmes. À l'inverse de la tendance à la spécialisation et à l'uniformisation, l'agroécologie vise à promouvoir et gérer la biodiversité, en s'appuyant sur les services écosystémiques associés au « fond écosystémique » vu comme un bien commun (Ostrom, 1990). Cela peut amener à changer les modes d'organisation, de décision, et les pratiques en développant une gestion collective des espaces et des ressources. L'agroécologie est également mobilisée pour penser la transition vers des systèmes alimentaires durables (Stassart et al., 2012). L'agroécologie et l'écologie industrielle sont envisagées comme deux voies complémentaires par certains auteurs (Thomas et al., 2014) pour satisfaire la demande croissante en produits d'origine animale, tout en préservant les ressources naturelles, en augmentant l'autonomie des exploitations ou des territoires, et en valorisant les savoir-faire locaux.

\section{Ce que des chercheurs en sciences agronomiques retiennent de ces notions pour traiter des interactions entre filières agricoles et territoires}

L'analyse des convergences et divergences entre ces notions met en exergue différents points de débat qu'il nous semble important de prendre en considération afin de pouvoir mieux interagir entre chercheurs et avec les acteurs de territoires. Il s'agit de se positionner, à partir des différentes façons de les considérer, sur: les dimensions des interactions entre filières et territoires (du fait de différentes façons de considérer les filières et les territoires) ; les dynamiques de ces interactions; les liens à l'action par rapport aux interrogations des acteurs de territoires.

\subsection{Différentes dimensions des interactions entre filières et territoires : ancrage, dépendance, empreinte}

Les différentes approches, vues dans le premier paragraphe, ont en commun de considérer la dimension matérielle des relations entre activités humaines et écosystèmes, en identifiant le «métabolisme» des activités, c'est-à-dire la circulation des flux de matières et d'énergie, ainsi que les stocks. L'objectif est de réduire l'usage des ressources non ou coûteusement renouvelables, et les «empreintes environnementales » des activités humaines, par le (re)bouclage des cycles de la matière. Par contre, ces notions divergent entre elles par leur façon de considérer les filières agricoles, les territoires et leurs interactions (Tab. 1).

Nous pouvons distinguer, à l'instar de Cerceau et al. (2014), trois visions des interactions entre filières et territoires :

- une vision considérant essentiellement le support biophysique (dimension matérielle) de ces interactions. Elle mobilise un corpus méthodologique au service de la description des flux de matières et d'énergie et d'une évaluation de leur empreinte environnementale ;

- une vision de ces interactions reposant sur un système d'acteurs territorialisé (dimensions organisationnelle et identitaire). Elle s'appuie sur des outils de description des jeux d'acteurs, et une analyse des formes d'ancrage et d'impact territorial de ces interactions ;

- une vision de ces interactions comme système complexe (dimensions matérielle, organisationnelle et identitaire), articulant l'espace biophysique, les acteurs qui le composent et la représentation qu'ils se font de celui-ci. Elle s'appuie sur la mise en œuvre d'outils de diagnostics capables de faire émerger des intérêts communs, notamment par une mise en lumière du métabolisme de ces interactions, de leurs empreintes et des interdépendances à d'autres territoires (ressources et/ou opérateurs), pour mieux penser les formes d'ancrage à mettre en place. C'est dans cette dernière vision, englobant les deux autres, que nous inscrivons notre proposition de cadre d'analyse.

Et de ces différentes approches, nous retenons trois dimensions des interactions entre filières et territoires : les formes d'ancrage des filières aux territoires, de dépendance des territoires pour la production agricole et ses valorisations, vis-à-vis de ressources et opérateurs extérieurs, et d'empreinte des filières pour les territoires. Chacune de ces dimensions se décline par rapport à des aspects socioéconomiques et environnementaux.

\subsection{Dynamiques des interactions entre filières et territoires}

Les approches présentées divergent aussi par les dynamiques et temporalités considérées (Tab. 1).

Nous pouvons distinguer deux visions des dynamiques des interactions entre filières et territoires : une vision circulaire du temps, considérant les cycles de la matière, de production, de vie des produits, la circulation des flux, qui met plutôt l'accent sur le fonctionnement au moment présent, en vue d'améliorer ce fonctionnement; une vision dynamique des interactions et de leurs évolutions, passées comme futures, qui resitue le fonctionnement au moment présent, à la fois dans ses fondements historiques et ses perspectives, en vue de comprendre dans quelles dynamiques s'inscrit le fonctionnement actuel pour pouvoir mieux agir dessus, et imaginerd'autres trajectoires. C'est dans cette deuxième vision, non exclusive de la première, que nous situons notre proposition de cadre d'analyse.

\subsection{Liens à l'action}

Il est intéressant de remarquer que certaines notions ont retenu «écologie» et d'autres «économie» dans leur intitulé. Cela laisse voir des entrées et visions différentes (Tab. 1). 


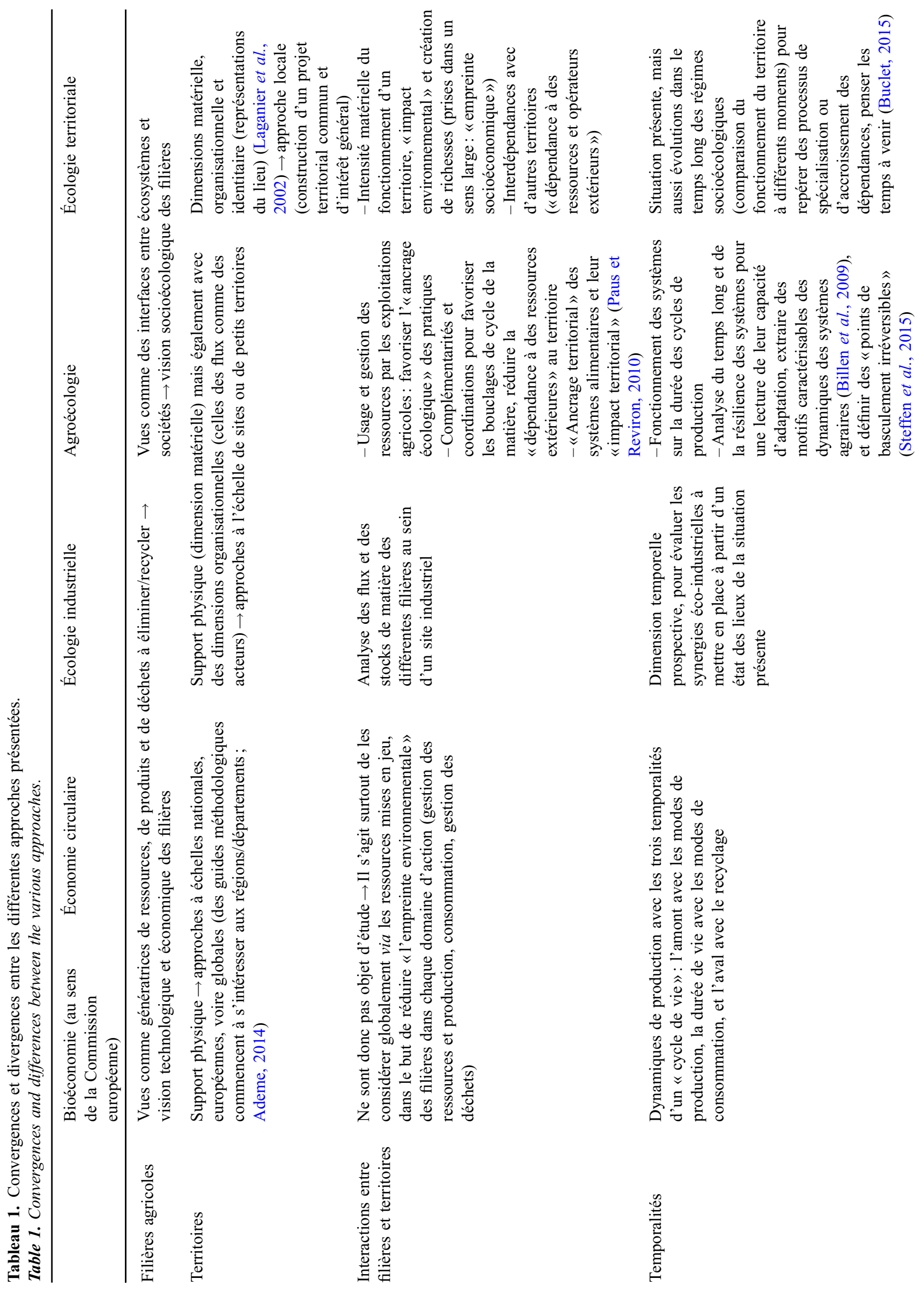


Mais ce n'est pas strict, étant donnée l'évolution, dans la pratique, de certaines notions.

Nous pouvons distinguer différentes visions du lien à l'action: une vision normative (performances économiques et environnementales à atteindre) qui met les questions d'optimisation et de technologies au cœur des solutions (Cerceau et al., 2014), en jouant plutôt sur l'efficience ou la substitution de facteurs (Hill et MacRae, 1996). Cette vision considère essentiellement la dimension matérielle des interactions entre filières et territoires (éventuellement organisationnelle) et une conception circulaire du temps; une vision participative et constructive qui replace l'acteur au centre des réflexions (Ehrenfeld, 2004b) et qui met les questions de biens communs, de définition des intérêts communs, de reconception en profondeur du système (Hill et MacRae, 1996) au cœur des démarches, et qui prend en compte l'ensemble des dimensions des interactions, et les dynamiques d'évolutions. C'est dans cette dernière vision que s'inscrit notre proposition de cadre d'analyse.

\section{Proposition d'un cadre d'analyse des interactions entre filières et territoires}

Le cadre d'analyse que nous proposons pour traiter la problématique des interactions entre filières agricoles et territoires, repose sur les trois précédentes entrées (dimensions des interactions, dynamiques des interactions, liens à l'action).

\subsection{Les formes d'interaction entre filières et territoires}

Ces formes d'interaction renvoient aux trois dimensions mises en évidence à partir des différentes approches, et permettent de préciser un diagnostic de la situation, à partir d'une lecture:

- des formes d'ancrage des filières agricoles aux territoires. Cela permet de resituer la façon dont les filières :

- s'appuient sur l'usage des ressources locales et les valorisent (propriétés des écosystèmes, ressources socioéconomiques ou culturelles),

- sont reliées entre elles ou à d'autres activités localement (bouclages de flux, offre d'un bouquet de produits et services);

- des formes de dépendance des territoires, pour la production agricole et ses valorisations, vis-à-vis de ressources et d'opérateurs exogènes, ou d'autres territoires. Saisir ces formes de dépendance permet de compléter l'analyse des formes d'ancrage, par celles des intrants utilisées par les filières, et des centres de décision et stratégies des opérateurs échappant à la gouvernance locale. Il peut en effet se trouver des filières ancrées localement d'un point de vue socioéconomique mais qui le sont peu écologiquement, et qui peuvent être fortement dépendantes d'intrants venant d'ailleurs. On peut également trouver des productions très ancrées écologiquement parlant, mais dépendantes de filières pilotées à une échelle nationale ou internationale et donc également fragilisées par de potentielles décisions prises en dehors de toute considération stratégique pour le territoire; 
- des formes d'empreinte des filières agricoles pour les territoires ou de la production agricole du territoire pour le reste du monde. Mesurer les empreintes de ces formes d'interaction entre filières et territoires permet enfin d'en proposer une évaluation environnementale et socioéconomique. Il peut s'agir de mesurer les ressources mises en jeu, les impacts, ou encore les services « rendus » ou richesses créées, soit par les filières agricoles pour les territoires, soit de la production agricole d'un territoire et de sa valorisation pour le reste du monde.

\subsection{Les évolutions au cours du temps}

Saisir les évolutions des interactions entre filières agricoles et territoires permet de resituer la réalité observable dans une dynamique, à la fois dans ses fondements historiques et ses perspectives. Elle permet à la fois de comprendre la façon dont les filières agricoles modifient leur environnement, et réciproquement, et comment cela accroît leur résilience et celle des territoires où elles se situent, ou au contraire produit des conditions de non-durabilité.

\subsection{Les conditions et modalités de construction d'une gouvernance territoriale des filières agricoles}

Les points 4.1 et 4.2 peuvent permettre de nourrir les réflexions avec les acteurs des territoires, en vue d'initier des dynamiques de changement dans une perspective de développement territorial durable, concernant notamment la gestion des ressources et la construction de «richesses territoriales ». Cela peut permettre d'identifier les freins et leviers à une (re)-territorialisation des filières agricoles. Il s'agit d'envisager de quelle façon l'appréhension des dynamiques observées peut aider à construire des transitions ad hoc. Une des questions est celle des réorganisations des activités humaines et des nouvelles formes de coopération entre acteurs à construire, pour rendre possible le découplage entre le développement économique des filières agricoles et la croissance de la consommation des ressources, à l'échelle des territoires.

\section{Discussion : intérêts et limites de l'approche}

\section{1 ... dans le champ de l'agronomie}

Notre proposition de cadre d'analyse donne à voir différentes dimensions des interactions entre filières et territoires, renvoyant à différents périmètres aux imbrications multiples. Or, chaque périmètre ne renvoie pas aux mêmes enjeux ni aux mêmes acteurs, n'est pas régi par les mêmes règles et systèmes d'action et de décision. L'entrée territoriale peut permettre de réconcilier la sphère de la production et de la valorisation des produits, et celle de la gestion environnementale de l'espace, souvent dissociées. Notre proposition vise ainsi à permettre de saisir la diversité et la complexité de ces formes d'interaction, ce qu'il faut comprendre si on veut notamment transformer le métabolisme de ces interactions et re-territorialiser les filières en vue d'un développement plus durable (Buclet, 2015). L'ambition du cadre d'analyse proposé est d'élargir le champ de l'analyse de l'ancrage territorial des filières à partir de celui de l'usage des écosystèmes locaux, et des dimensions complémentaires sur les formes de dépendance et d'empreinte. Il vise à permettre l'analyse des formes de territorialisation des filières agricoles, mais surtout des différentes dimensions de cette territorialisation (en termes d'ancrage, de dépendance, d'empreinte, à la fois environnemental et socioéconomique).

Les limites de ce cadre sont qu'il n'explicite pas encore bien le lien avec le maillon de la consommation, alors que des travaux en montrent l'influence (Billen et al., 2012). L'analyse des relations entre filières, à l'échelle des territoires, reste également à approfondir. Des travaux commencent à ébaucher des pistes de réflexions, qu'il s'agisse de bouclages de flux (Nowak et al., 2015) ou d'intégration entre cultures et élevages à l'échelle de territoires (Moraine et al., 2016); mais ces interactions (via des flux biophysiques, de travail ou d'équipements, économiques, via des liens de proximités entre acteurs plus ou moins formalisés, via des formes de concurrence ou de synergie) restent largement à caractériser à l'échelle territoriale, d'autant plus que le développement de certaines filières peut se faire au détriment d'autres (Gedouin et al., 2015). Ce sont autant de perspectives pour la recherche agronomique, en collaboration avec d'autres disciplines. Cela requiert de fédérer une communauté de recherche, débordant la seule discipline agronomique, ce qu'on aborde plus avant dans un autre article de ce même numéro (Madelrieux et al., 2017). Mais cela nécessite également un dialogue explicite avec les acteurs, pour repositionner les ressources et leurs systèmes de gestion dans les territoires. C'est alors un méta-programme de recherche-action à l'échelle des territoires qu'il s'agit de déployer.

\section{2 ... à l'échelle des territoires}

Pour développer des coordinations entre acteurs des filières et des territoires et des modes de gouvernance favorisant la gestion durable des ressources d'un territoire (Ostrom, 1990), l'effort de recherche devrait aussi s'orienter sur des dispositifs favorisant les interactions entre chercheurs et acteurs (Hubert et al., 2004). Clarifier les notions et proposer un cadre de questionnement et d'analyse est, pour nous, un premier pas pour formaliser des démarches plus opérationnelles. Ce cadre vise à poser les bases pour l'élaboration d'outils nécessaires à des réflexions de prospective territoriale. Ces outils doivent permettre de caractériser les modes de production et de valorisation des activités agricoles, et les filières associées et leurs relations, de mesurer leurs empreintes environnementale et socioéconomique pour le territoire, et de les resituer dans leurs dynamiques. Ils doivent pouvoir servir d'«objets intermédiaires » dans un processus de conception participative (Vinck, 2009), pour réfléchir et discuter collectivement des synergies et des compromis à développer, et permettre la construction d'une gouvernance territoriale des filières agricoles. On peut citer l'expérience d'une recherche-action ayant permis la re-territorialisation d'une filière laine dans le Pays de Saugues en Haute-Loire (Grison et al., 2014). Pour autant, les expérimentations peinent à se concrétiser. 
Mais, tout au long de telles démarches, il s'agit de veiller à acquérir une meilleure connaissance des facteurs et conditions de réussite dans l'usage des concepts et méthodes avec les acteurs, et de leurs effets réels. L'analyse de leur utilisation pour stimuler la participation des acteurs dans l'élaboration de scénarios de changement est en effet rare (Bousquet et Le Page, 2004). Pourtant il y a un réel enjeu à approfondir les méthodologies afin de parvenir à une étude au plus près du territoire « vécu» (Debuisson, 2014).

\section{Conclusion}

Nous avons illustré l'existence d'une pluralité de notions visant à penser ensemble l'économie et l'écologie des activités humaines. Elles convergent sur la dimension matérielle des activités humaines. Elles divergent sur les cadres de pensée et finalités, sur les façons de considérer les filières agricoles, les territoires et leurs interactions. Nous avons montré également la forte proximité entre agroécologie et écologie territoriale, dans leur cadre de pensée, même si les champs d'application sont différents. Ces deux notions reposent sur les mêmes fondamentaux : la critique des approches des activités économiques qui ne se soucient pas des limites de l'environnement (Figuière et al., 2014), et un changement de paradigme. En effet, au lieu de chercher à réduire les composantes non durables des systèmes (améliorations de produits et de procédés mais qui restent dommageables pour l'environnement - Ehrenfeld, 2004b), ces approches visent à construire une durabilité «forte» des systèmes. Pour cela, il s'agit de (re)créer du lien entre des activités, des ressources, et des acteurs, proches géographiquement, mais qui n'ont pas forcément de liens, dans une redéfinition de l'intérêt général. Elles vont plus loin que le seul recours à des bouclages de flux, ou à la substitution d'intrants chimiques par des intrants biologiques (Duru et al., 2016) comme voies d'un développement durable, et incitent à repenser les liens entre activités humaines et propriétés des écosystèmes, notamment avec la notion de métabolisme. Pour autant, la notion de métabolisme se décline différemment selon les approches (métabolisme industriel, urbain, territorial, socioéconomique) et fait encore débat (voir par exemple, pour le métabolisme industriel : Wassenaar, 2015). Certaines des notions abordées peuvent être considérées comme insuffisamment abouties du point de vue de la gestion des ressources naturelles et des écosystèmes (Erb, 2012) ou de l'analyse des jeux d'acteurs. Mais nous avons cherché à tirer parti de cette pluralité, et de son décryptage, pour élaborer un cadre d'analyse des interactions entre filières et territoires, basé sur les questions des formes d'interaction (ancrage, dépendance, empreinte), leurs évolutions dans le temps et les conditions et modalités de construction d'une gouvernance territoriale des filières agricoles.

\section{Références}

Ademe. 2014. Guide méthodologique du développement des stratégies régionales d'économie circulaire en France. ADEME, 89 p. Disponible sur http://www.ademe.fr/guide-methodologiquedeveloppement-strategies-regionales-deconomie-circulaire-france.

Altieri MA. 1987. Agroecology: the scientific basis of alternative agriculture. USA: Westview Press.
Barles S. 2011. L'écologie territoriale: qu'est-ce que c'est? In: Ecotech\&tool Conference, 30 novembre-2 décembre 2011, Montpellier.

Beaurain C, Brullot S. 2011. L'écologie industrielle comme processus de développement territorial: une lecture par la proximité. Revue d'Économie Régionale et Urbaine 2: 313-340.

Billen G, Barles S, Garnier J, Rouillard J, Benoit P. 2009. The foodprint of Paris: long-term reconstruction of the nitrogen flows imported into the city from its rural hinterland. Regional Environnemental Change 9(1): 13-24.

Billen G, Garnier J, Thieu V, Silvestre M, Barles S, Chatzimpiros P. 2012. Localising the nitrogen imprint of the Paris food supply: the potential of organic farming and changes in human diet. Biogeosciences 9: 607-616.

Bousquet F, Le Page C. 2004. Multi-agent simulations and ecosystem management: a review. Ecological Modelling 176: 313-332.

Brullot S, Maillefert M, Joubert J. 2014. Stratégies d'acteurs et gouvernance des démarches d'écologie industrielle et territoriale. Développement durable et territoires 5(1). Disponible sur http:// developpementdurable.revues.org/10082.

Buclet N. 2011. Écologie industrielle et territoriale. Stratégies locales pour un développement durable. Villeneuve d'Ascq: Presses universitaires du Septentrion.

Buclet N, ed. 2015. Essai d'écologie territoriale. L'exemple d'Aussois en Savoie. Paris : CNRS Editions.

Cerceau J, Junqua G, Gonzalez C, Laforest V, Lopez-Ferber M. 2014. Quel territoire pour quelle écologie industrielle ? Contribution à la définition du territoire en écologie industrielle. Développement durable et territoires 5(1). Disponible sur http://developpementdu rable.revues.org/10179.

Costanza R 1989. What is ecological economics? Ecological Economics 1: 1-7.

Costanza R, Cumberland JH, Daly H, Goodland R, Norgaard RB, Kubiszewski I, et al. 2015. An introduction to ecological economics, 2nd ed. Boca Raton: CRC Press, Taylor \& Francis Group.

Debuisson M. 2014. Les modes d'interaction pour une dynamique territoriale soutenable : un apport à l'écologie territoriale. Thèse de Doctorat, université de technologie de Troyes.

Diemer A, Labrune S. 2007. L'écologie industrielle : quand l'écosystème industriel devient un vecteur du développement durable. Développement Durable et Territoire. DOI:10.4000/ developpementdurable.4121.

Duru M, Therond O, Roger-Estrade J, Richard G. 2016. Agriculture models at the crossroads of farming systems, food systems and territorial dynamics. In: 12th European IFSA Symposium, 12-15 July 2016, Harper Adams University, UK.

Ehrenfeld J. 2004a. Searching for sustainability: no quick fix. Reflections: the SoL Journal 5(8): 137-149.

Ehrenfeld J. 2004b. Industrial ecology: a new field or only a metaphor? Journal of Cleaner Production 12: 825-831.

Emelianoff C. 2000. L'écologie urbaine entre science et urbanisme. Quaderni 43: 85-99.

Emelianoff C. 2008. La problématique des inégalités écologiques, un nouveau paysage conceptuel. Écologie \& politique 1(35): 19-31.

Erb KH. 2012. How a socio-ecological metabolism approach can help to advance our understanding of changes in land-use intensity. Ecological Economics 76: 8-14.

Erkman S. 2004. Vers une écologie industrielle, $2^{\mathrm{e}}$ ed. Paris : Charles Leopold Mayer Ed.

European Commission. 2012. Innovating for sustainable growth: a bioeconomy for Europe. Brussels: European Commission. Available from http://ec.europa.eu/research/bioeconomy/pdf/ 201202_innovating_sustainable_growth_en.pdf. 
Fader M, Gerten D, Krause M, Lucht W, Cramer W. 2013. Spatial decoupling of agricultural production and consumption: quantifying dependences of countries on food imports due to domestic land and water constraints. Environ Res Lett 8. DOI:10.1088/17489326/8/1/014046.

Fares M, Magrini MB, Triboulet P. 2012. Transition agro-écologique, innovation et effets de verrouillage: le rôle de la structure organisationnelle des filières. Le cas de la filière blé dur française. Cahiers Agricultures 21: 34-45.

Figuière C, Boidin B, Diemer A. 2014. Économie politique du développement durable. Louvain-la-Neuve: Ed. de Boeck.

Fischer-Kowalski M, Haberl H. 2007. Socioecological transitions and global change: trajectories of social metabolism and land use. UK: Edward Elgar Publishing.

Francis C, Lieblein G, Gliessman S, Breland TA, Creamer N, Harwood R, et al. 2003. Agroecology: the ecology of food systems. Journal of Sustainable Agriculture 22: 99-118.

Frosch RA, Gallopoulos N. 1989. Strategies for manufacturing. Scientific American 261(3): 144-152.

Gedouin M, Saravia A, Tourrand JF. 2015. Élevage à l'herbe et développement du soja en Uruguay: transformations d'un modèle pastoral dominant. Fourrages 221: 47-56.

Georgescu-Roegen N. 1979. La décroissance: entropie, écologie, économie. Paris : Éditions du sang de la terre.

Grinevald J. 1993. L'utilisation rationnelle de l'énergie. La révolution bioéconomique de Nicholas Georgescu-Roegen. À propos de la première conférence internationale de bioéconomie. Disponible sur http://www.akademia.ch/ sebes/textes/1993/93JGrinevald.html.

Grison JB, Fournier M, Rieutort L, Masurier D, Berthold R. 2014. Développement d'un projet de coopération territoriale pour la structuration d'un pôle laine en Pays de Saugues, Clermont Ferrand. Rapport de recherche CERAMAC. Disponible sur https:// halshs.archives-ouvertes.fr/halshs-00978241v1.

Hénin S. 1967. Les acquisitions techniques en production végétale et leurs applications. Économie rurale 74(1): 31-44.

Hill SB, MacRae RJ. 1996. Conceptual frameworks for the transition from conventional to sustainable agriculture. Journal of Sustainable Agriculture 7(1): 81-87.

Hubert B, Moulin CH, Roche B, Pluvinage J, Deffontaines JP. 2004. Quels dispositifs pour conduire des recherches en partenariat? L'intervention d'une équipe de recherche au Pays basque intérieur. Économie rurale 279: 33-52.

Laganier R, Villalba B, Zuindeau B. 2002. Le développement durable face au territoire : éléments pour une recherche pluridisciplinaire. Développement durable et territoires. DOI:10.4000/developpe mentdurable. 774 .

Madelrieux S, Buclet N, Lescoat P, Moraine M. 2017. Caractériser les formes d'interaction entre filières agricoles et territoires: quelles méthodes? Cahiers Agricultures 26: 24002.

McGinnis MD, Ostrom E. 2014. Social-ecological system framework: initial changes and continuing challenges. Ecology and Society 19(2). Available from http://doi.org/10.5751/ES-06387-190230.

Manouvrier E. 2008. Une filière en recomposition et ses incidences territoriales : l'endive dans la France du Nord. Revue d'Économie Régionale \& Urbaine 2: 211-224.
Meadows DH, Meadows DL, Randers J, Behrens WW. 1972. The limits to growth. New York: Universe Books.

Millenium Ecosystem Assessment (MEA) 2005. Ecosystems and human well-being: general synthesis. Island Press.

Missemer A. 2013. Nicholas Georgescu-Roegen, pour une révolution bioéconomique. Lyon: ENS Editions.

Muchnik J, Sanz Cañada J, Torres Salcido G. 2008. Systèmes agroalimentaires localisés : état des recherches et perspectives. Cahiers Agricultures 17: 513-519.

Nesme T, Withers P. 2016. Sustainable strategies towards a phosphorus circular Economy. Nutr Cycl Agroecosyst 4: 259-264.

Nowak B, Nesme T, David C, Pellerin S. 2015. Nutrient cycling in organic farming is related to diversity in farm types at the local level. Agriculture, Ecosystems and Environment 204: 17-26.

Ostrom E. 1990. Governing the commons: the evolution of institutions for collective action. Cambridge University Press.

Pauliuk S, Hertwich EG. 2015. Socioeconomic metabolism as paradigm for studying the biophysical basis of human societies. Ecological Economics 119: 83-93.

Paus M, Reviron S. 2010. Mesure de l'impact territorial d'initiatives agroalimentaires. Enseignement de deux cas suisses. Économie rurale 315: $28-45$.

Rastoin JL, Ghersi G. 2010. Le système alimentaire mondial. Concepts et méthodes, analyses et dynamiques. Versailles : Éditions Quae.

Rees WE. 1992. Ecological footprints and appropriated carrying capacity: what urban economics leaves out. Environment and Urbanization 4(2): 121-130.

Schaller N. 2013. L'agroécologie: des définitions variées, des principes communs. Centre d'études et de prospectives 59.

Stassart PM, Baret P, Grégoire JC, Hance T, Mormont M, Reheul D, et al. 2012. L'agroécologie : trajectoire et potentiel. Pour une transition vers des systèmes alimentaires durables. In : Van Dam D, Nizet J, Streith M, Stassart PM, eds. Agroécologie entre pratiques et sciences sociales. Dijon : Educagri édition, pp. 25-51.

Steffen W, Richardson K, Rockström J, Cornell SE, Fetzer I, Bennett EM, et al. 2015. Planetary boundaries: guiding human development on a changing planet. Science 347. DOI:10.1126/ science. 1259855.

Thomas M, Fortun-Lamothe L, Jouven M, Tichit M, Gonzalez-Garcia E, Dourmad JY, Dumont B. 2014. Agro-écologie et écologie industrielle : deux alternatives complémentaires pour les systèmes d'élevage de demain. Inra Prod Anim 27(2): 89-100.

Van der Ploeg JD. 2008. The new peasantries. Struggles for autonomy and sustainability in an era of empire and globalization. London: Earthscan.

Vinck D. 2009. De l'objet intermédiaire à l'objet-frontière. Vers la prise en compte du travail d'équipement. Revue d'anthropologie des connaissances 3(1): 51-72.

Wassenaar T. 2015. Reconsidering industrial metabolism. From analogy to denoting actuality. Journal of Industrial Ecology 19(5): 715-727.

Wezel A, Bellon S, Doré T, Francis C, Vallod D, David C. 2009. Agroecology as a science, a movement and a practice. A review. Agronomy for Sustainable Development 29: 503-515.

Citation de l'article : Madelrieux S, Buclet N, Lescoat P, Moraine M. 2017. Écologie et économie des interactions entre filières agricoles et territoire: quels concepts et cadre d'analyse? Cah. Agric. 26: 24001. 